\title{
The contemporary status of robotic intracorporeal neobladder
}

\author{
Fouad Maqboul', Johnraj Kishore Raja Thinagaran', Zach Dovey ${ }^{1,2}$, Peter Wiklund ${ }^{2}$ \\ 'Department of Urology, Ashford and St. Peter's Hospital NHS Trust Foundation, Chertsey, Surrey KT16 OPZ, UK. \\ ${ }^{2}$ Department of Urology, Mount Sinai Hospital, New York, NY 10029, USA.
}

Correspondence to: Dr. Zach Dovey, Department of Urology, Mount Sinai Hospital, Icahn Medical School 1, Gustav L. Levy Place, New York, NY 10029, USA. E-mail: zachary.dovey@mountsinai.org

How to cite this article: Maqboul F, Thinagaran JKR, Dovey Z, Wiklund P. The contemporary status of robotic intracorporeal neobladder. Mini-invasive Surg 2021;5:44. https://dx.doi.org/10.20517/2574-1225.2021.54

Received: 22 Apr 2021 First Decision: 22 Jun 2021 Revised: 8 Jul 2021 Accepted: 27 Jul 2021 First online: 3 Aug 2021

Academic Editor: Riccardo Autorino Copy Editor: Xi-Jun Chen Production Editor: Xi-Jun Chen

\begin{abstract}
Robotic intracorporeal neobladder (RIN) is increasingly the modality of choice for intracorporeal urinary diversion in high-volume Robotic Urology centers. This article details the modern technique of RIN, explains specific tips and tricks to facilitate timely operative progression as well as weighs the outcomes from recently published series. An OVID/EMBASE database search was done using keywords: robotic, cystectomy, intracorporeal neobladder, orthotopic, and intracorporeal urinary diversion. The inclusion criteria were original studies on Robot-Assisted Radical Cystectomy (RARC) with RIN series, available in full text in English, published over the last ten years with a specific analysis of oncological and functional outcomes. Pooled data analysis of the 10 studies included shows $80 \%$ of patients had organ-confined disease ( $\leq \mathrm{pT} 2), 1.86 \%$ of patients had positive surgical margin, median lymph node yield of 23 nodes (IQR $=7.5$ ), and cancer-specific survival rate of $78 \%$ (range $72 \%-100 \%$ ) over a mean follow up of 27.43 months (range 13-37 months). Functionally, the median day continence rate is $81.5 \%$, night continence rate is $61 \%$, and rate of return to spontaneous sexual activity is $33.5 \%$. This compares favorably with outcomes of The International Robotic Cystectomy Consortium - Extracorporeal Urinary Diversion data and data from open radical cystectomy (ORC) neobladder series with long term follow up. High-volume robotic centers have successfully introduced programs for RARC, with RIN demonstrating its safety and feasibility. Their results suggest potential to improve perioperative and functional outcomes over ORC. Moreover, under mentorship, surgeons can learn the technique of RARC and RIN without these outcomes being significantly affected.
\end{abstract}


Keywords: Neobladder, cystectomy, robotic, intracorporeal, orthotopic

\section{INTRODUCTION}

Surgical treatment of muscle-invasive (MIBC) and high-risk non-muscle-invasive bladder cancer is uniformly recommended. However, despite the emergence and global spread of robotic techniques, there is still some controversy over the suggested advantage of robotic-assisted over open approaches. Moreover, the choice of intracorporeal urinary diversion (ICUD), which is technically challenging, adds to this debate, further polarised by robotic intracorporeal neobladder (RIN), the most difficult technical option of all. Nevertheless, robotic cystectomies remain steadfast in their belief that their approach is superior despite the lack of clear scientific proof by randomized controlled trials. It is reflected in the widespread adoption of RARC and RIN by high-volume robotic urological centers. Between 2015 and 2018, 70\% in North America and $50 \%$ in Europe of Radical Cystectomy (RCs) performed were done robotically ${ }^{[1]}$, with an increase in ICUD from $9 \%$ to $97 \%$ between 2005 and $2015^{[2]}$. This increase in ICUD was primarily accounted for by centers performing intracorporeal ileal conduit, which increased from $2 \%$ to $81 \%$, rather than RIN, which only increased from $7 \%$ to $17 \%{ }^{[2]}$. RIN is regarded as one of the most technically challenging procedures in robotic urology. Its steep learning curve (LC), lack of indisputable evidence of patient benefit, and the economic drawbacks of prolonged theater time may all explain why its widespread adoption has been slower. Nevertheless, as urological centers gain experience with clearly defined mentorship programs and published outcomes with increasingly long follow-up in appropriately selected patients, RIN has shown to provide equivalent oncological outcomes and long-term quality of life (QoL) compared to the open approach. This review will detail the current techniques for RIN, highlight patient selection, discuss the results of published series to date as well as key ongoing trials and outline some of the related issues, including Enhanced Recovery after Surgery (ERAS) protocols, the LC for the procedure and economics.

\section{Patient selection}

Patient selection is crucial to a successful operative outcome and will be influenced by surgeon experience, clinical factors affecting the decision to undertake a robotic procedure, and specific patient factors related to the choice of neobladder itself. Generally, the ideal patient for Robot-Assisted Radical Cystectomy (RARC) will be under 75 years of age, slim with a BMI of less than 30, have a T2 tumor without locally advanced disease, have good performance status with minimal co-morbidities, and no history of prior abdominal radiation or surgery ${ }^{[3]}$. As surgeons gain experience, these guidelines will be flexed, but whatever the surgeon experience is, patients with BMI > 35, complex cardio-respiratory co-morbidities and prior abdominal or vascular surgery, pelvic trauma or radiotherapy, and locally advanced disease will prove challenging. Even with these factors in mind, further consideration is required when selecting patients for RIN, such as the physiological and cognitive requirements to adapt to a neobladder that can significantly impact the outcome and patient's quality of life. The main contraindications for orthotopic neobladder are renal and liver impairment, tumor invading the prostatic apex and bladder neck (which would result in a positive intraoperative urethral margin). In addition, there are relative contraindications as the lack of patient's motivation or cognitive impairment, preventing adherence to the bladder-training program, physical limitations hinder the ability to intermittently self-catheterize, and a damaged urethral sphincter that would result in severe incontinence ${ }^{[4]}$.

\section{Technique}

To date in the literature, the majority of published series have used the Studer U modified neobladder, the largest series of which was from Karolinska detailing the technique and outcomes of 158 patients since its introduction in $2003^{[5]}$. Other techniques used for RIN include modified Hautmann W, the Padua, the Y technique, the Florence, the Vescica Ileale Padovana, the Pyramid pouch, and the Camey Reservoir ${ }^{[6]}$. This 
review will focus on the Karolinska modified Studer $U$ technique, which is the procedure that the authors have the most experience in and was also the most common technique used in our series review [Table 1].

\section{Patient preparation, set up, and trocar positioning}

Most high-volume robotic centers will engage in ERAS protocols for robotic cystectomy and ICUD ${ }^{[7]}$. Regarding preoperative patient advice, ERAS protocols now recommend written and oral counselling and education on the nature of the procedure and postoperative care as well as preoperative medical optimization. Mechanical bowel preparation can be avoided; suitable patients should be encouraged to have preoperative carbohydrate loading. In the $24 \mathrm{~h}$ prior to surgery, they should adopt a low residue diet, with solids and clear fluids for 6 and $2 \mathrm{~h}$ respectively ${ }^{[]]}$.

In the operating room (OR), the patient is placed under general anesthesia and in the lithotomy position with the operating table at maximum Trendelenburg position. Pneumatic calf compression is applied to reduce the risk of deep vein thrombosis, the patient's arms are fixed along their sides, and a body warmer is used over the thorax to prevent hypothermia. Antibiotic prophylaxis with broad-spectrum antibiotics should be given at induction, and low molecular weight heparin is given in the early postoperative period and continued for 4 weeks post-surgery.

Trocar positioning is a crucial part of the procedure; the operation can proceed smoothly by setting up the robotic arms and instrument positions. With the Da Vinci Xi system, the $8 \mathrm{~mm}$ camera port is placed first, 1-2 $\mathrm{cm}$ to the left of the midline and 3-5 $\mathrm{cm}$ above the umbilicus. Next, two further robotic $8 \mathrm{~mm}$ ports are placed $8-10 \mathrm{~cm}$ right and left of the midline at the level of the umbilicus. The left-sided assistant port is $2-3 \mathrm{~cm}$ above and medial to the anterior superior iliac spine (ASIS), as well as $8-10 \mathrm{~cm}$ away from the leftsided robotic port. It is a $15 \mathrm{~mm}$ port that allows the bowel stapler to pass through during the bowel work and anastomosis. At other times, a robotic $8 \mathrm{~mm}$ port with the instrument can be telescoped through it as the "fourth arm". The final two ports are $12 \mathrm{~mm}$ assistant ports, the first symmetrically between the camera and right robotic port, and far right, $8-10 \mathrm{~cm}$ from the right robotic port, approximately $2-3 \mathrm{~cm}$ superior and medial to the right $\mathrm{ASIS}^{[8]}$. Next, we discuss the neobladder formation and skipping cystectomy part.

\section{THE STUDER U MODIFIED NEOBLADDER (SEE FIGURES 1-18 AND THE “TIPS AND TRICKS" SECTION)}

The original description of the Studer $U$ orthotopic neobladder has been modified according to Wiklund and Poulakis ${ }^{[8]}$ for robotic intracorporeal reconstruction. Although other robotic centers may have their own modifications to Studer's original technique, the fundamental steps are the same ${ }^{[9]}$. After trocar placement, the RARC and extended pelvic lymph node dissection (EPLND) are carried out before the RIN. For the purposes of standardization and education, the RIN procedure is then broken down into the following modules:

Demarcation of the neobladder bowel segment [Figure 1]: $50 \mathrm{~cm}$ of ileum is identified, at least $25 \mathrm{~cm}$ away from the ileo-cecal junction, and brought down into the pelvis in a U shape. The "tip" of the U is the site of the urethro-ileal anastomosis (UIA). The right ileal limb above the UIA will be $10 \mathrm{~cm}$ in length, and the left ileal limb will be $40 \mathrm{~cm}$ in length. Bringing the ileum down to the urethra is generally easier in females.

Urethro-ileal anastomosis [Figures 2 and 3]: in keeping with the Rocco principle and to allow a tension-free anastomosis, the posterior aspect of ileal serosa is sutured to the sub-urethral tissues. Following this, a $2 \mathrm{~cm}$ incision is made through the ileum into the lumen, and the formal anastomosis is completed using doubleneedle 3-0 monofilament synthetic absorbable suture (Biosyn). The first sutures are placed on the urethra in 
Table 1. Robotic intracorporeal neobladder study series: the perioperative outcomes

\begin{tabular}{|c|c|c|c|c|c|c|c|c|c|}
\hline Study & $\begin{array}{l}\text { Technique } \\
\text { of RIN }\end{array}$ & $\begin{array}{l}\text { Number of } \\
\text { patients had } \\
\text { RARC }\end{array}$ & $\begin{array}{l}\text { Number of } \\
\text { patients had } \\
\text { RIN }\end{array}$ & $\begin{array}{l}\text { Median OT } \\
(\mathrm{min}) \text { in } \\
\text { RIN }\end{array}$ & $\begin{array}{l}\text { EBL } \\
(\mathrm{mL}) \text { in } \\
\text { RIN }\end{array}$ & $\begin{array}{l}\text { Patients had } \\
\text { transfusion in } \\
\text { RIN }\end{array}$ & $\begin{array}{l}\text { Number of events of } \\
\text { complication Clavien-Dindo } \\
\geq \mathrm{III} \text { at } 30 \text { days in RIN }\end{array}$ & $\begin{array}{l}\text { Number of events of } \\
\text { complication Clavien-Dindo } \\
\geq \mathrm{III} \text { at } 90 \text { days in RIN }\end{array}$ & $\begin{array}{l}\text { Median length of } \\
\text { hospital stay } \\
\text { (days) in RIN }\end{array}$ \\
\hline Collins et al. ${ }^{[39]} 2014$ & Studer U & 147 & 80 & 420 & $\mathrm{n} / \mathrm{a}$ & $\mathrm{n} / \mathrm{a}$ & $\mathrm{n} / \mathrm{a}$ & $\mathrm{n} / \mathrm{a}$ & $\mathrm{n} / \mathrm{a}$ \\
\hline Goh et al. ${ }^{[40]} 2012$ & Studer U & 15 & 8 & 450 & 225 & 3 & 2 & 2 & 8 \\
\hline Hosseini et al. ${ }^{[5]} 2020$ & Studer U & 158 & 158 & 363 & 300 & $\mathrm{n} / \mathrm{a}$ & 35 & 10 & 8 \\
\hline $\begin{array}{l}\text { Obrecht et al. }{ }^{[20]} \\
2020\end{array}$ & $\begin{array}{l}\text { Modified } \\
\text { Studer }\end{array}$ & 12 & 12 & 575 & 600 & $\mathrm{n} / \mathrm{a}$ & $\mathrm{n} / \mathrm{a}$ & $\mathrm{n} / \mathrm{a}$ & $\mathrm{n} / \mathrm{a}$ \\
\hline Tyritzis et al. ${ }^{[41]} 2013$ & Studer U & 70 & 70 & 420 & 500 & 3 & 22 & 13 & 9 \\
\hline Desai et al. ${ }^{[42]} 2014$ & Studer U & 132 & 132 & 456 & 430 & 6 & 20 & 17 & 10.6 \\
\hline Tuderti et al. ${ }^{[13]} 2020$ & Padua & 11 & 11 & 255 & $\mathrm{n} / \mathrm{a}$ & $\mathrm{n} / \mathrm{a}$ & 0 & $\mathrm{n} / \mathrm{a}$ & 7 \\
\hline $\begin{array}{l}\text { Schwentner et al. }{ }^{[43]} \\
2015\end{array}$ & Studer U & 62 & 62 & 476 & 385 & $\mathrm{n} / \mathrm{a}$ & 16 & 16 & 17 \\
\hline Gu et al. ${ }^{[44]} 2020$ & Studer U & 12 & 12 & 419 & 400 & 8 & 1 & 1 & 14.5 \\
\hline Jonsson et al. ${ }^{[23]} 2011$ & $\begin{array}{l}\text { Modified } \\
\text { Studer }\end{array}$ & 45 & 36 & 480 & 625 & $\mathrm{n} / \mathrm{a}$ & 3 & 5 & 9 \\
\hline
\end{tabular}

RIN: Robotic intracorporeal neobladder; RARC: robot-assisted radical cystectomy; OT: operating time; EBL: estimated blood loss.

to out either side of 6 o'clock, and the suturing is completed circumferentially over a 22 Fr catheter, out to in on the ileal side, and in to out on the urethral side.

Isolation of neobladder bowel segment and re-anastomosis of the bowel [Figure 4]: once the UIA is complete, as described above, the bowel is divided with Endo-GIA ${ }^{\mathrm{m}}$ Laparoscopic staplers which are passed through the "fourth arm" $15 \mathrm{~mm}$ port, once the robotic instrument has been removed. It is done $10 \mathrm{~cm}$ above the UIA for the right ileal neobladder limb and $40 \mathrm{~cm}$ above the UIA for the left ileal neobladder limb. The ileum is then re-anastomosed using both a $60 \mathrm{~mm}$ and a $45 \mathrm{~mm}$ cartridge, finally closing the upper aspect of the anastomosed bowel with another $60 \mathrm{~mm}$ cartridge.

Detubularization of the ileal neobladder limbs and formation of the posterior plate [Figures 5 and 6 ]: both limbs of the neobladder are opened over the suction instrument, except for $10 \mathrm{~cm}$ of the proximal aspect of the left ileal limb, which forms the "chimney". Once detubularized, stay sutures are placed to bring the medial sides of the top of the right ileal neobladder limb to the medial aspect of the left ileal neobladder limb, just at the bottom of the chimney. Two more stay sutures are placed. First, $10 \mathrm{~cm}$ below the chimney, the medial aspect of the left ileal neobladder limb is sutured to the upper aspect of the ileum just above the UIA. It leaves an open U-shaped loop of $20 \mathrm{~cm}$ of the left ileal neobladder limb on the left side of the pelvis. The final stay suture is placed at its most lateral aspect, bringing the inner aspect of the upper and lower parts of this loop together. With the stay sutures in place, the different parts of the posterior plate are 


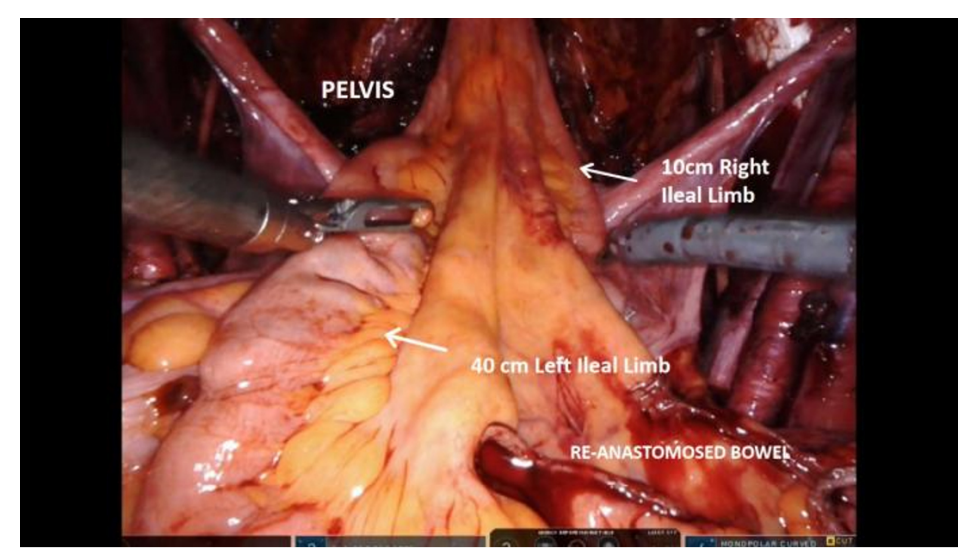

Figure 1. Demarcation of Neobladder bowel segment.

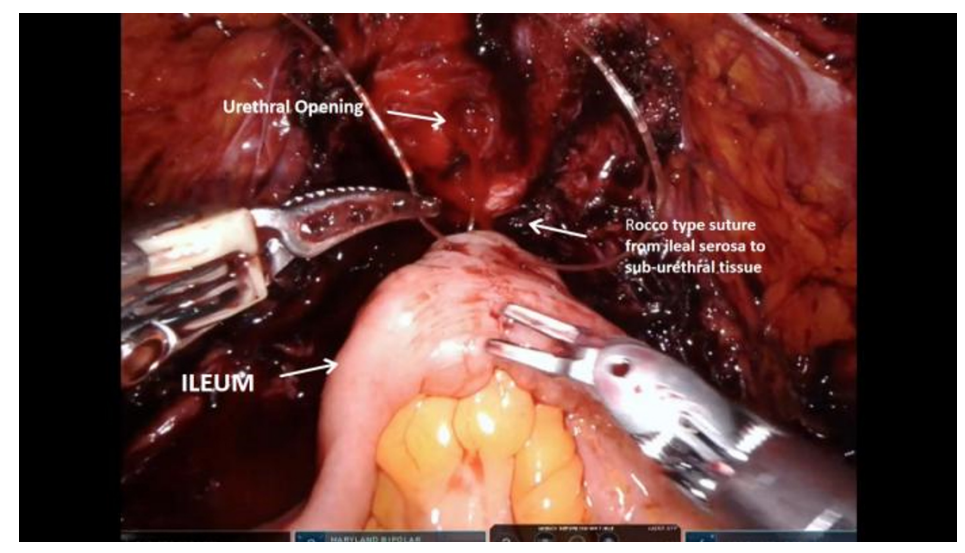

Figure 2. Urethro-ileal anastomosis A.

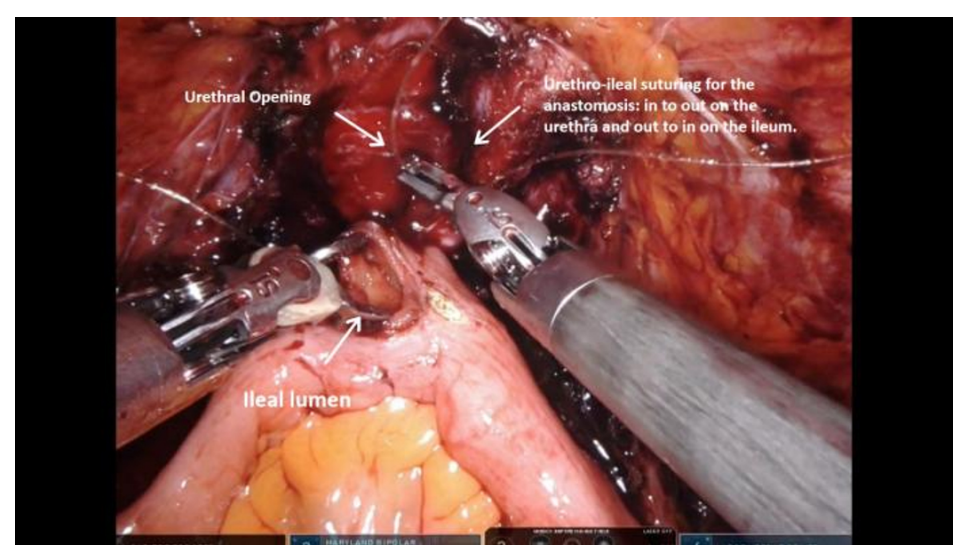

Figure 3. Urethro-ileal anastomosis B.

clearly defined and closed with a 3/0 V-loc suture.

Anterior neobladder closure [Figures 7-9]: once the posterior plate has been constructed, the anterior part of the neobladder is closed with a similar 3/0 V-Loc suture; starting by folding the left side of the neobladder 


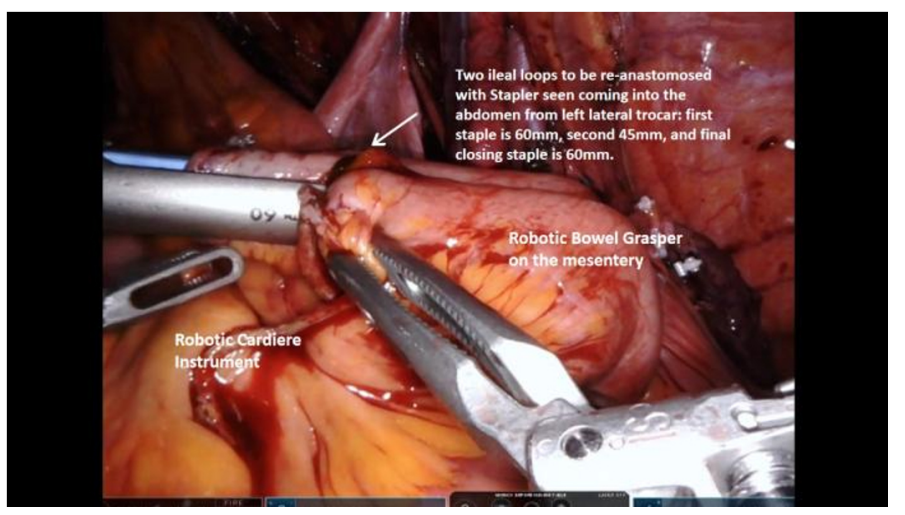

Figure 4. Isolation of neobladder bowel segment and re-anastomosis of the bowel.

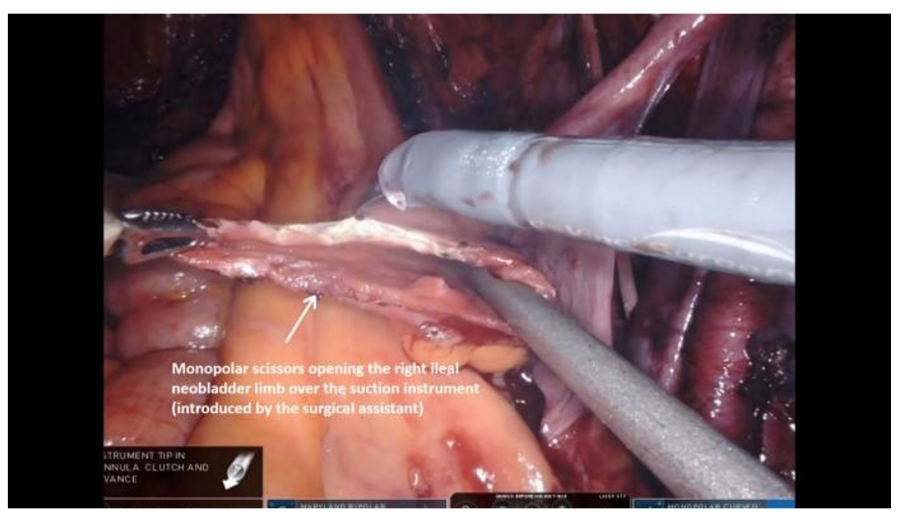

Figure 5. Detubularisation of the ileal neobladder limbs and formation of the posterior plate A.

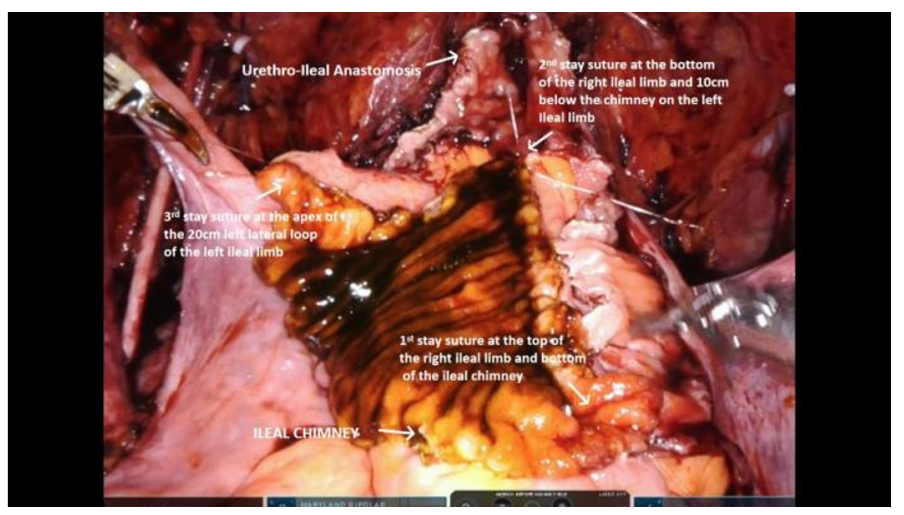

Figure 6. Detubularisation of the ileal neobladder limbs and formation of the posterior plate B.

at the lateral aspect of the left ileal limb lateral loop up to the base of the chimney (see Figures 7-9), and then continuing distally with a running V-loc suture down to the UIA. At its most proximal aspect, the neobladder is initially left open, in order to pass the ureteric stents.

Uretero-ileal anastomosis, stent insertion, and completion of neobladder closure (Figures 10-18): a Wallacetype uretero-ileal anastomosis is the used technique. Following the dissection of the ureters at the beginning of the cystectomy, the left ureter is passed below the sigmoid mesentery to the right side of the pelvis. Both 


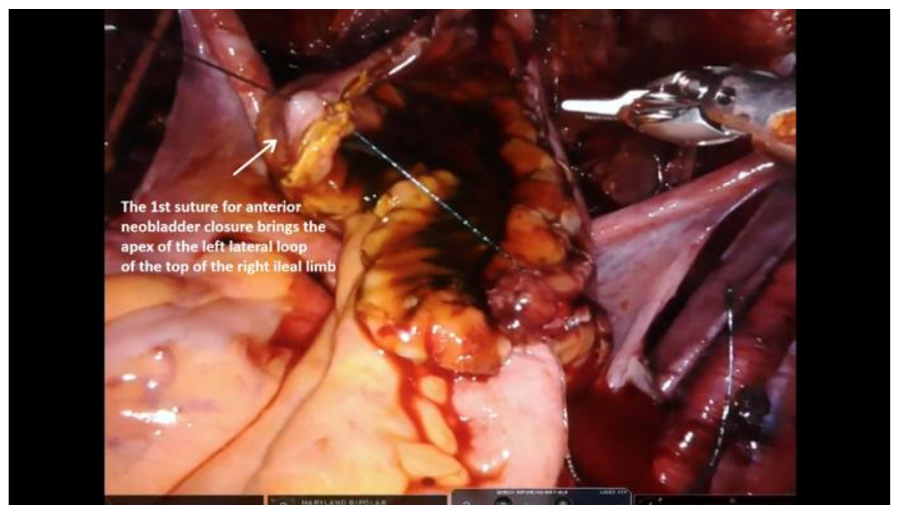

Figure 7. Anterior neobladder closure A.

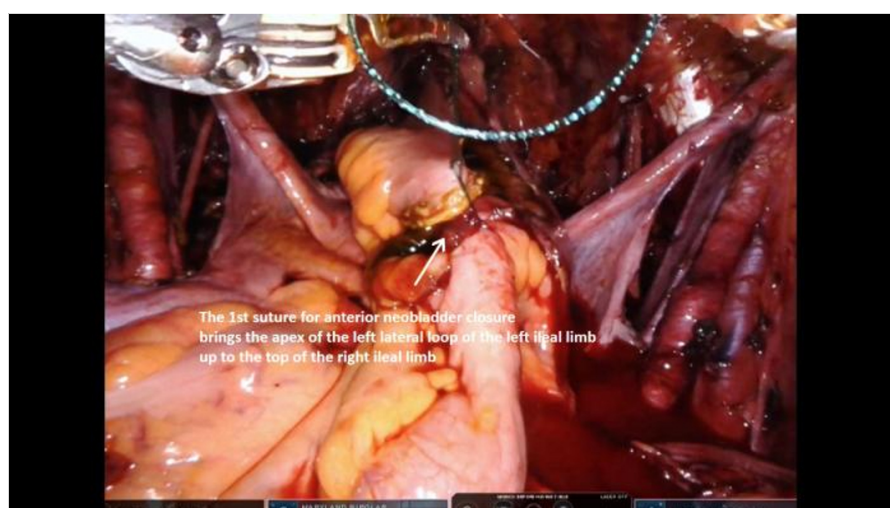

Figure 8. Anterior neobladder closure B.

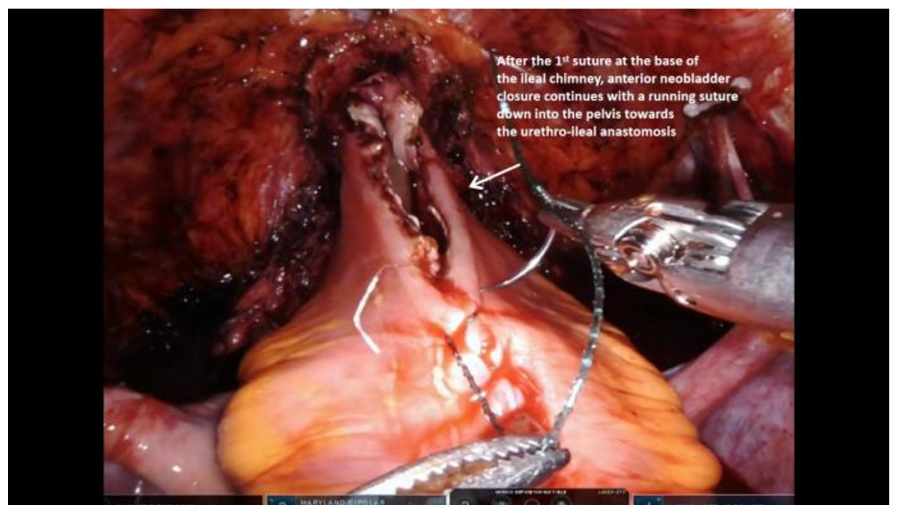

Figure 9. Anterior neobladder closure C.

ureters are then lifted vertically adjacent to each other, and the posterior plate of the Wallace anastomosis is done with an absorbable 4-0 monofilament suture [Figures 11-14]. A nephrostomy puncture needle is passed through the suprapubic area in the midline of the abdomen, through which both stents are passed [Figure 10]. Each one is then pulled through the opening in the anterior wall of the neobladder by the fourth arm, out through the ileal chimney, and inserted down each ureter. In keeping with the Wallace approach, the ureters are then anastomosed circumferentially with the open ileal chimney again using 4-0 absorbable monofilament suture, and the residual opening in the anterior wall of the neobladder is closed around the 


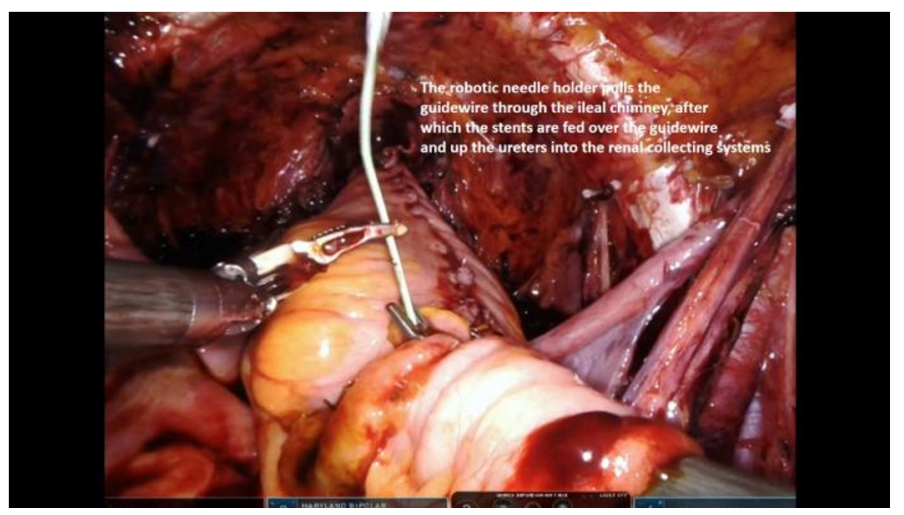

Figure 10. Stent insertion.

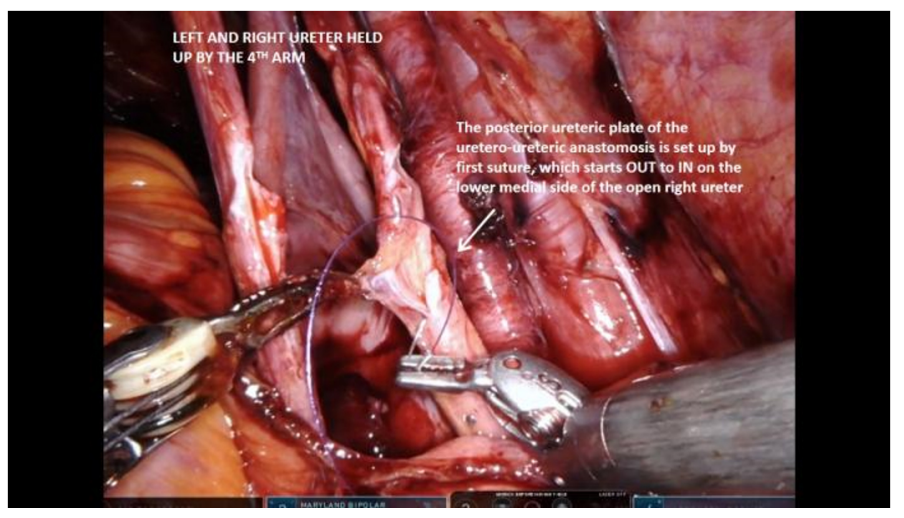

Figure 11. Posterior plate of Wallace uretero-ureteric anastomosis A.

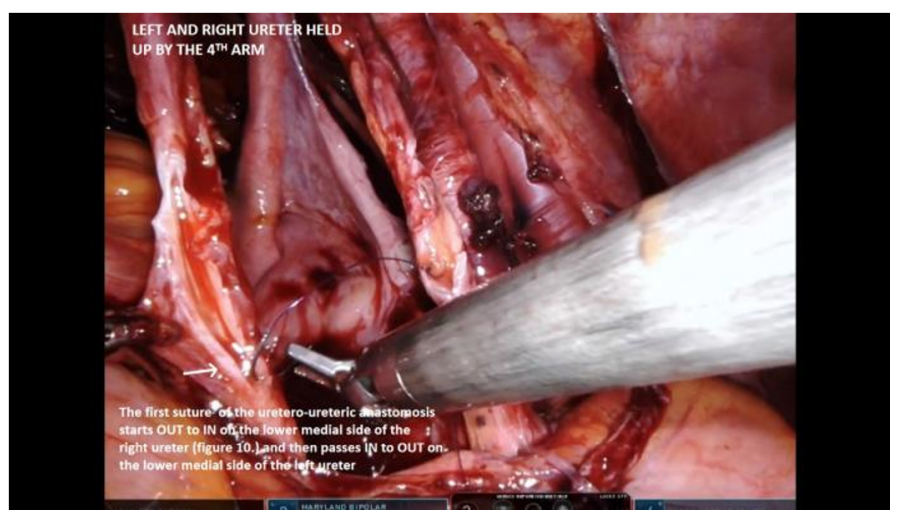

Figure 12. Posterior plate of Wallace uretero-ureteric anastomosis B.

stents with 3-0 absorbable monofilament sutures [Figures 15-17]. Once closed, the neobladder reservoir can be tested for leakage by inflation with approximately $120 \mathrm{cc}$ of normal saline injected through the $22 \mathrm{Fr}$ urethral catheter [Figure 18].

\section{Review of outcomes; evidence acquisition (see Tables 1-4 and Appendix 1)}

To study comparative outcomes of RIN with respect to open intracorporeal neobladder, a literature search on OVID and EMBASE database was done while using keywords: robotic, cystectomy, intracorporeal 


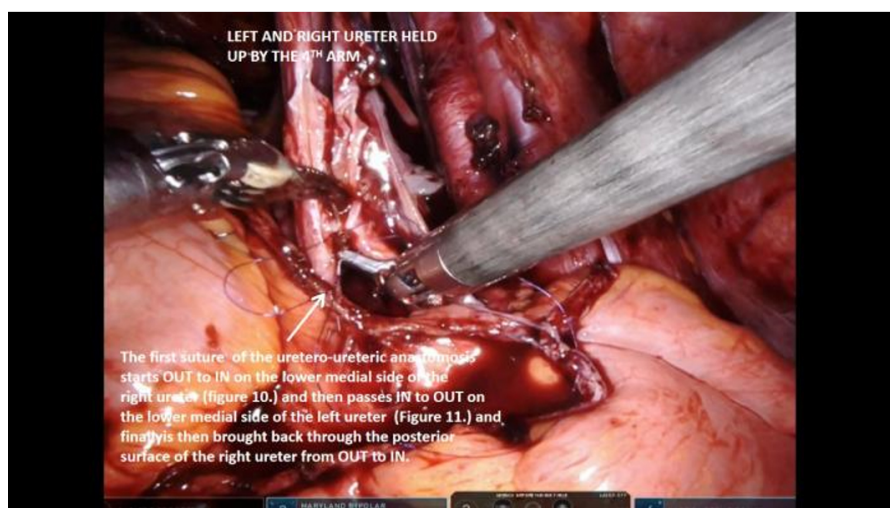

Figure 13. Posterior plate of Wallace uretero-ureteric anastomosis C.

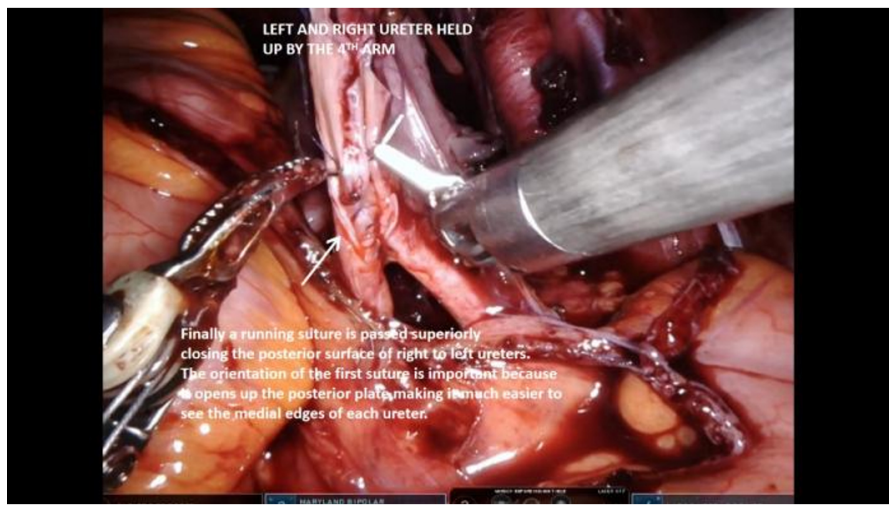

Figure 14. Posterior plate of Wallace uretero-ureteric anastomosis D.

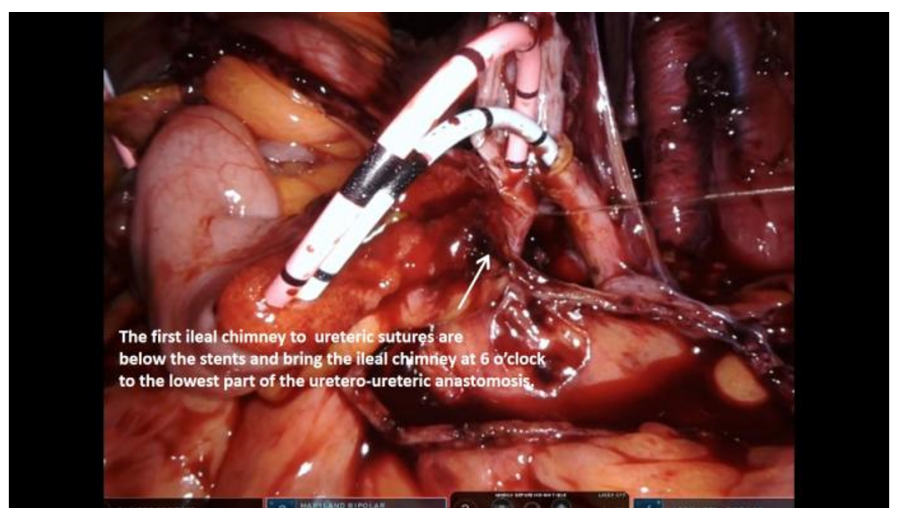

Figure 15. Ileo-ureteric anastomosis A.

neobladder, orthotopic, and intracorporeal urinary diversion. For evidence acquisition, inclusion and exclusion criteria are illustrated in Appendix 1. Inclusion criteria were original studies on RARC with RIN series, available in full text in English, published over the last 10 years, and with a specific analysis of oncological and functional outcomes. Overall, 10 studies were included and reviewed (see Table 1). 


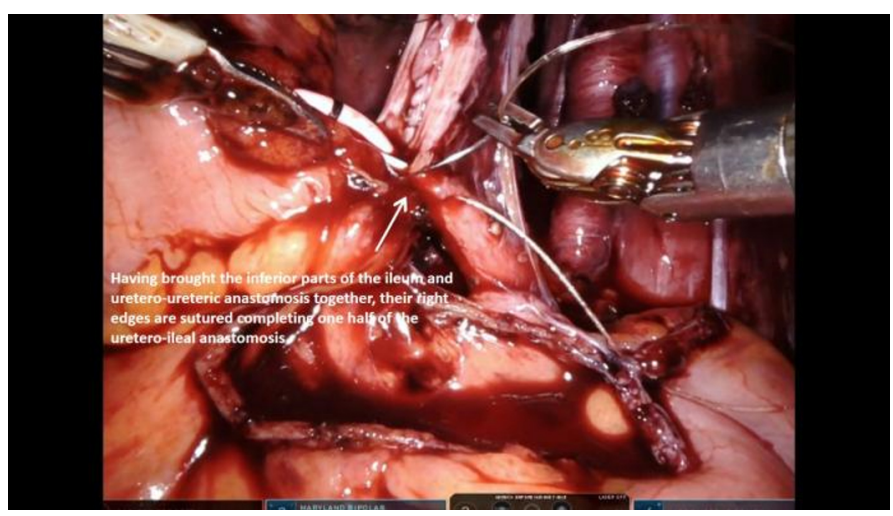

Figure 16. Ileo-ureteric anastomosis B.

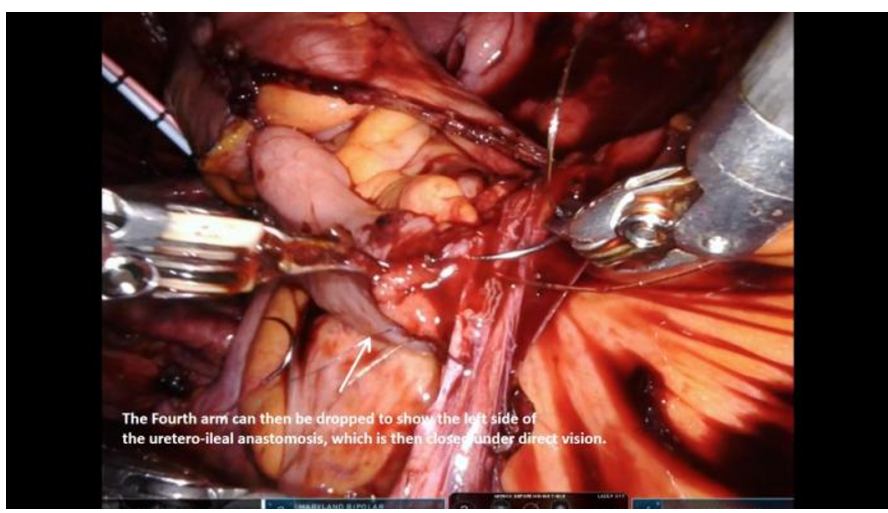

Figure 17. Ileo-ureteric anastomosis C

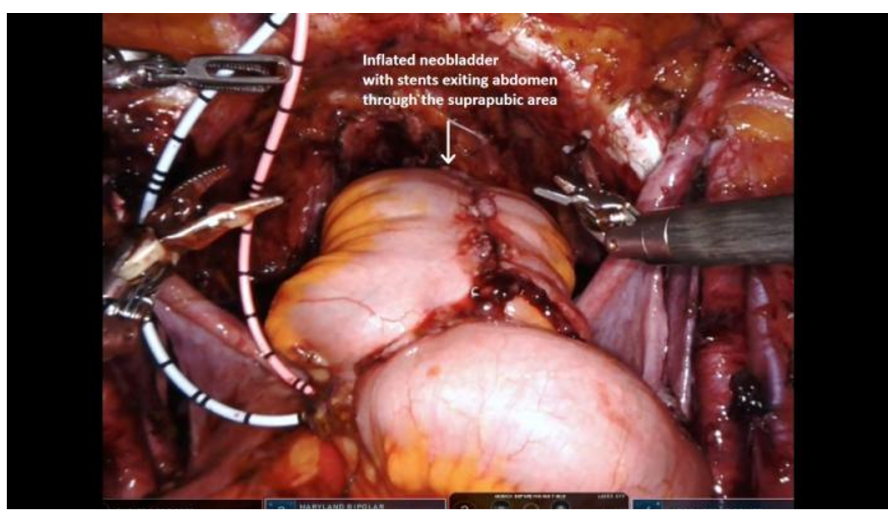

Figure 18. Inflation of neobladder.

RIN is the logical evolution of the minimally invasive approach for RARC, and it is recognized as its most technically challenging part. Moreover, most of the complications of the procedure are caused by this reconstruction. Studies focused on learning curves for RIN are discussed in more detail below, but, interestingly, it is not only operating times (OTs) that fall but also the rate of high-grade complications as centers gain experience ${ }^{[5]}$. Although oncological outcomes data may seem more dependent on the cystectomy and extended lymph node dissection, the choice of RIN may also influence oncological outcomes by prolonged OTs or incomplete resection of tumor at the urethral margin. Nevertheless, apart 
Table 2. Robotic intracorporeal neobladder study series: the oncological outcomes

\begin{tabular}{|c|c|c|c|c|c|c|c|}
\hline Study & $\begin{array}{l}\text { Number of patients had } \leq \mathrm{pT} 2 \\
\text { (organ confined) postoperative } \\
\text { in RIN }\end{array}$ & $\begin{array}{l}\text { Number of patients had } \\
\text { positive surgical } \\
\text { margins in RIN }\end{array}$ & $\begin{array}{l}\text { Lymph node } \\
\text { yield (number) }\end{array}$ & $\begin{array}{l}\text { Mean follow up } \\
\text { (months) for } \\
\text { cancer }\end{array}$ & $\begin{array}{l}\text { Patients with the } \\
\text { disease who are still } \\
\text { alive at follow up }\end{array}$ & $\begin{array}{l}\text { Est. Disease } \\
\text { survival rate DSR\% } \\
\text { at } 5 \text { years }\end{array}$ & $\begin{array}{l}\text { Est. Overall } \\
\text { Survival rate OSR\% } \\
\text { at } 5 \text { years }\end{array}$ \\
\hline Collins et al. ${ }^{[39]} 2014$ & $\mathrm{n} / \mathrm{a}$ & $\mathrm{n} / \mathrm{a}$ & $\mathrm{n} / \mathrm{a}$ & $\mathrm{n} / \mathrm{a}$ & $\mathrm{n} / \mathrm{a}$ & $\mathrm{n} / \mathrm{a}$ & $\mathrm{n} / \mathrm{a}$ \\
\hline Goh et al. ${ }^{[40]} 2012$ & 6 & 0 & 55 & $\mathrm{n} / \mathrm{a}$ & $\mathrm{n} / \mathrm{a}$ & $\mathrm{n} / \mathrm{a}$ & $\mathrm{n} / \mathrm{a}$ \\
\hline Hosseini et al. ${ }^{[5]} 2020$ & 129 & 2 & 23 & 34 & 114 & 70 & 71 \\
\hline $\begin{array}{l}\text { Obrecht et al. }{ }^{[20]} \\
2020\end{array}$ & 11 & $\mathrm{n} / \mathrm{a}$ & $\mathrm{n} / \mathrm{a}$ & $n / a$ & $\mathrm{n} / \mathrm{a}$ & $\mathrm{n} / \mathrm{a}$ & $\mathrm{n} / \mathrm{a}$ \\
\hline Tyritzis et al. ${ }^{[41]} 2013$ & 57 & 1 & 21 & 30 & 62 & 80.7 & 88.9 \\
\hline Desai et al. ${ }^{[42]} 2014$ & 109 & 1 & 29 & 25 & 95 & 71 & 72 \\
\hline Tuderti et al. ${ }^{[13]} 2020$ & 7 & 0 & 26 & 28 & 11 & 100 & 100 \\
\hline $\begin{array}{l}\text { Schwentner et al. }{ }^{[43]} \\
2015\end{array}$ & 38 & 4 & 23 & 37 & 52 & 76 & 71 \\
\hline Gu et al..$^{[44]} 2020$ & 9 & 0 & 11 & 13 & 10 & $\mathrm{n} / \mathrm{a}$ & $\mathrm{n} / \mathrm{a}$ \\
\hline Jonsson et al. ${ }^{[23]} 2011$ & 35 & 1 & 19 & 25 & 31 & 84 & $\mathrm{n} / \mathrm{a}$ \\
\hline
\end{tabular}

RIN: Robotic intracorporeal neobladder.

Table 3. Robotic intracorporeal neobladder study series: the functional outcomes

\begin{tabular}{|c|c|c|c|c|}
\hline Study & $\begin{array}{l}\text { Number of patients had daytime } \\
\text { continence }^{\star}\end{array}$ & $\begin{array}{l}\text { Number of patients had nighttime } \\
\text { continence }\end{array}$ & $\begin{array}{l}\text { Number of patients achieved functionl erection } \\
\text { (not aided) }\end{array}$ & $\begin{array}{l}\text { Mean follow up for continence } \\
\text { (months) }\end{array}$ \\
\hline Collins et al. ${ }^{[39]} 2014$ & $\mathrm{n} / \mathrm{a}$ & $\mathrm{n} / \mathrm{a}$ & $\mathrm{n} / \mathrm{a}$ & 12 \\
\hline Goh et al. ${ }^{[40]} 2012$ & 6 & $\mathrm{n} / \mathrm{a}$ & $\mathrm{n} / \mathrm{a}$ & 3 \\
\hline Hosseini et al. ${ }^{[5]} 2020$ & $\mathrm{n} / \mathrm{a}$ & $\mathrm{n} / \mathrm{a}$ & $\mathrm{n} / \mathrm{a}$ & $\mathrm{n} / \mathrm{a}$ \\
\hline $\begin{array}{l}\text { Obrecht et al. } \\
2020\end{array}$ & 12 & 9 & $\mathrm{n} / \mathrm{a}$ & 6 \\
\hline Tyritzis et al. ${ }^{[41]} 2013$ & 48 & 40 & 12 & 12 \\
\hline Desai et al. ${ }^{[42]} 2014$ & 62 & $\mathrm{n} / \mathrm{a}$ & $\mathrm{n} / \mathrm{a}$ & 6 \\
\hline Tuderti et al. ${ }^{[13]} 2020$ & 10 & 9 & 8 & 12 \\
\hline $\begin{array}{l}\text { Schwentner et al. }{ }^{[43]} \\
2015\end{array}$ & 54 & 34 & 27 & 37 \\
\hline Gu et al. ${ }^{[44]} 2020$ & 9 & 8 & 2 & 6 \\
\hline Jonsson et al. ${ }^{[23]} 2011$ & 30 & 24 & 15 & 12 \\
\hline
\end{tabular}

${ }^{*}$ Continence is defined as using 0-1 pads. Study excluded as follow up was less than 6 months. 73 patients only were followed for functional outcome. 
Table 4. Robotic intracorporeal neobladder study series: result of pooled data analysis for all outcomes

\begin{tabular}{|c|c|}
\hline Outcome & Median/Rate (interquartile range/calculation) \\
\hline \multicolumn{2}{|l|}{ Peri-operative outcome } \\
\hline Median OT (min) in RIN & $435(I Q R=57)$ \\
\hline Median EBL ( $\mathrm{mL}$ ) in RIN & $415(I Q R=207.5)$ \\
\hline Mean transfusion rate in RIN & $8.73 \%(20 / 229)$ \\
\hline Mean complication Clavien-Dindo $\geq$ III rate at 30 days in RIN & $20.25 \%(99 / 489)$ \\
\hline Mean complication Clavien-Dindo $\geq \mathrm{III}$ rate at 90 days in RIN & $13.39 \%(64 / 478)$ \\
\hline Median length of hospital stay (days) in RIN & $9(\mathrm{IQR}=4.5)$ \\
\hline \multicolumn{2}{|l|}{ Oncology outcome } \\
\hline Mean Post-operative organ confined disease ( $(\mathrm{pT} 2)$ in RIN & $80.04 \%(401 / 405)$ \\
\hline Mean Positive surgical margins in RIN & $1.86 \%(9 / 485)$ \\
\hline Median lymph node yield (number) & $23(I Q R=7.5)$ \\
\hline Mean follow up (months) & 27.43 (range: 13-37) \\
\hline Calculated mean cancer survival rate CSR\% & $77.96 \%(375 / 481)$ \\
\hline Estimated median disease survival rate DSR\% & $78.35 \%(I Q R=13)$ \\
\hline Estimated median overall survival rate OSR\% & $72 \%(I Q R=23.4)$ \\
\hline \multicolumn{2}{|l|}{ Functional outcome (studies with follow up at least for 6 months) } \\
\hline Mean daytime continence rate & $81.52 \%(225 / 276)$ \\
\hline Mean nighttime continence rate & $61.08 \%(124 / 203)$ \\
\hline Mean spontaneous sexual activity/potency rate & $33.51 \%(64 / 191)$ \\
\hline
\end{tabular}

IQR: Interquartile range; RIN: robotic intracorporeal neobladder; OT: operating time; EBL: estimated blood loss.

from the obvious functional advantages, there is also the potential for less bowel manipulation, less blood and fluid loss, less hypothermia, and reduced ureteric trauma leading to lower ureteric stricture rates ${ }^{[1,10,1]}$. Although a small proportion of patients in the reviewed series underwent intracorporeal ileal conduits, the overwhelming majority OF $87.5 \%$ had RARC with RIN.

\section{Perioperative outcomes (see Table 1)}

Less blood and fluid loss has been suggested as an advantage for ICUD. Prior figures from the recent IRCC analysis of ICUD vs. ECUD, of which $21 \%$ vs. $23 \%$ were neobladders, found less estimated blood loss (EBL) for ICUD at $300 \mathrm{~mL} v s .350 \mathrm{~mL}$ and less transfusion at $5 \%$ vs. 13\%. The median EBL of the series reviewed is $415 \mathrm{~mL}$ (IQR = 207.5), suggesting RIN may be associated with higher EBL compared to intracorporeal or extracorporeal ileal conduit. Where assessed, the transfusion rates in the series reviewed varied significantly from $4.2 \%$ to $66.7 \%$, reflected in the variation in the numbers of patients and experience of the surgeons, and on pooled analysis, the transfusion rate is at $8.7 \%$. For the larger series from established centers, transfusion rates for RIN (4.35\% and 4.5\%) are less than those quoted for ECUD in the IRCC data $(13 \%)^{[1]}$. Long OTs have contributed to complication rates and, with prolonged Trendelenburg position, may give rise to specific complications such as compartment syndrome or posterior infarction of the optic nerve. The combined OT of extended lymph node dissection, with additional nerve-sparing in men, or pelvic organ sparing in women will necessarily add to the duration of the procedure, sometimes breaching the recommended OT of the Pasadena Consensus Panel (PCP) of $<7 \mathrm{~h}^{[12]}$. Nevertheless, the larger series from high-volume centers note a definite drop in OTs over the period of study, Hosseini et al.$^{[5]}$ suggesting a plateau of approximately $5 \mathrm{~h}$ after 80 cases. Tuderti et al. ${ }^{[13]}$ performing an Intracorporeal Padua Ileal Neobladder with pelvic organ sparing RARC in women achieved a mean OT of $255 \mathrm{~min}$. The median OT in Table 1 is $435 \mathrm{~min}(\mathrm{IQR}=57)$, just over the $7 \mathrm{~h}$ recommended by the PCP. Once again, the emphasis is on high volume centers with experienced surgical teams as well as standardization of the procedure to reduce OTs. Ninety-days high-grade complications rate from this series is $13.39 \%$, ranging from $6 \%$ to $26 \%$. It 
compares favorably with the ICUD and ECUD high-grade complication rates ( $21 \%$ and $24 \%$ respectively) from the IRCC data ${ }^{[1]}$, as well as those of large open series from high volume centers ${ }^{[14]}$. When analyzed, some RIN series found that high-grade gastrointestinal complications were particularly low, strongly supporting the suggestion that keeping the bowel intra-abdominally and reducing its manipulation is beneficial ${ }^{[5]}$.

\section{Oncological outcomes (see Table 2)}

The influence of RIN on oncological outcomes is important to establish, and it has been suggested to result from the pneumoperitoneum causing tumor seeding that changes the pattern of recurrence, the minimally invasive approach reducing lymph node yield, and possibly an increase in positive surgical margins with or without incomplete resection of tumor at the urethral margin ${ }^{[15]}$. It has not been born out in the published results that have shown non-inferiority of RARC with $\operatorname{RIN}^{[16,17]}$, and this is further supported by the results of this review. In Table 2, the mean positive surgical margin of 1.86\% (range 0\%-6.4\%), median lymph node yield of 23 nodes (IQR $=7.5$ ), and mean cancer-specific survival rates of $78 \%$ (range $72 \%-100 \%$ ) over mean follow up of 23.2 months (range 3-37 months) compares favorably with oncological outcomes from ORC and RARC with ECUD series ${ }^{[18,19]}$.

\section{Functional outcomes (see Table 3)}

While perioperative and oncological outcomes may be comparable for robotic and open approaches, naturally, the minimally invasive approach, analogous to potency and continence outcomes for roboticassisted prostatectomy, may have the potential to demonstrate superiority in functional outcomes. The magnified view and dexterity afforded by robotic assistance may allow more accurate dissection around the pelvic floor with nerve and pelvic organ preservation, notwithstanding QoL improvements from a more physiologically functioning neobladder. The small study of Obrecht et al. ${ }^{[20]}$ (see Tables 1-3) examined functional outcomes after RARC with RIN over 12 months follow up including urodynamic measurements, bladder capacity, and a QoL assessment. Their results showed good functional outcomes with a median bladder capacity of $400 \mathrm{~mL}$, which are close to the normal physiological bladder capacity and day continence rates of $100 \%{ }^{[20]}$. In this series, no patients required ISC, in keeping prior results of lower ISC rates for robotic neobladder than open neobladders ${ }^{[21,22]}$. Similarly, from Table 3 in this review, the day continence rate is $81.5 \%$ (range $68 \%-100 \%$ ) while the night continence rate is $61 \%$ (range $55 \%-82 \%$ ), where continence was defined as using 0-1 pad. These results compare favorably to open neobladder series that demonstrate daytime and nighttime continence rates of $80 \%-100 \%$ and $45 \%-90 \%$ respectively ${ }^{[2]}$. Generally, there is a paucity of potency or return to sexual activity outcome data in the literature after RIN. Jonsson et al. ${ }^{[23]}$ has reported potency of $88 \%$ of men who underwent RIN and nerve-sparing technique with potency defined as IIEF-5 $\geq 17$ or the ability to perform intercourse. Tuderti et al. ${ }^{[13]^{\prime}}$ s study focusing on female patients after pelvic organ sparing RARC with RIN, showed favorable functional outcomes with daytime and nighttime continence rates $90.9 \%$ and $86.4 \%$ respectively and $72 \%$ of patients returning to sexual activity at 12 months. Overall, for all series reviewed in Table 3, the mean potency or spontaneous return to sexual activity rate was $33.51 \%$ (range 16\%-72\%). Although some results may show a trend towards improved results for RIN, the claim of superiority of RIN over extracorporeal or open neobladder is difficult to make until results from better-designed studies support $\mathrm{it}^{[24]}$.

The early results of such studies are seen from Mastroianni et al. ${ }^{[25]}$, who recently reported an interim analysis from an ongoing randomized controlled trial between ORC vs. RARC with ICUD on Healthrelated Quality of Life after 1-year using patient-reported questionnaires from EORTC group, generic quality of life [QLQ-C30] and bladder cancer-specific instruments [QLQ-BLM30] questionnaires. Both approaches have comparable baseline QoL, as patients of two groups reported worsening physical 
functioning, body image, and sexual functioning. The ORC group reported higher gastrointestinal symptoms of flatulence, irregular bowel habits, and abdominal bloating, which result in delayed return to normal daily activities and impaired role functioning (occupational, social, and financial roles), whereas patients who had RARC tend to experience more impairment of urinary symptoms and problems. Further results are expected with longer follow up reflected by urodynamic studies in both groups ${ }^{[25]}$.

Simone et al. ${ }^{[26]}$ assessed outcomes for prostate capsule and seminal vesicle sparing cystectomies to assess alternative techniques to improve the functional outcome. The results of 2 -year follow-up on 20 patients who had organ-confined disease at TURB, negative urethral biopsy, and PSA $<4 \mathrm{ng} / \mathrm{mL}$ showed better continence and sexual function but worse oncological outcomes with a higher local recurrence rate of $20 \%$ and distant metastasis rate of $30 \%$. It suggests a more traditional approach to nerve-sparing when dissecting the prostate, including excising the seminal vesicles with the prostate, may be a better technical approach when aiming to maximize functional outcomes ${ }^{[26]}$.

A summary of pooled analysis and outcomes of the series review is in [Table 4].

\section{Trials}

Evidence from recent trials has contributed to the outcomes debate. The RAZOR trial demonstrated that RARC is non-inferior to ORC for oncological outcomes, although the urinary diversion approach was extracorporeal in this trial. The two-year progression-free survival was $72.3 \%$ in RARC vs. $71.6 \%$ in ORC with no significant differences in lymph node yield, positive surgical margins, complication rates, or QoL assessments ${ }^{[24]}$. The results of the iROC trial investigating RARC with ICUD (both ileal conduit and RIN) in comparison to ORC and ECUD are anticipated. For this study, participating surgeons must have completed at least 30 procedures of each, which will be close to the plateau of their learning curves. The study will report on oncological, perioperative, functional, and cost outcomes for RARC with ICUD and hopefully make a major contribution to the literature, potentially resolving some outstanding areas of doubt in the argument for RARC and ICUD vs. the open approach ${ }^{[27]}$.

\section{Specific tips and tricks for the Karolinska modified Studer U bladder (see Table 5)}

As has been highlighted, longer OR times may contribute to surgical trauma, influence postoperative complications, and mean prolonged Trendelenburg position, and negatively influence economics and cost analyses. Having a fastidious and reproducible step-by-step approach will allow smooth progression of the procedure and timely completion as well as faster progress up the learning curve for surgeons under mentorship. These tips and tricks highlighted in Table 5. aim to facilitate this process.

\section{Economics}

The question of economics is relevant to the widespread adoption of RARC and RIN as a form of urinary diversion. The intracorporeal orthotopic reconstruction may increase operative time but also has the potential to increase morbidity and complications, both of which may increase costs. Although the evidence is conflicting, and there may be institutional variation in cost-effectiveness, the general consensus is that RARC is more expensive than $\mathrm{ORC}^{[28]}$. Using Prisma Methodology to select relevant studies, a recent review examined segmental costs to breakdown where the additional cost for RARC lies, examining the results from a total of 11 series. Operating costs, which included surgeon fees and occupation of the OR, both heavily dependent on OR time, accounted for $63.1 \%-70.5 \%$ of overall RARC costs, which will likely further increase with the addition of RIN ${ }^{[28]}$. Interestingly, in an earlier study, Lee et al. ${ }^{[29]}$ highlighted differences in costs between Neobladder and Ileal conduit after RARC, finding RARC and ileal conduit had a cost advantage over ORC of $\$ 4846$, which was reversed to $-\$ 1966$ if neobladder was done. Of note, for this study, all urinary diversions were extracorporeal, although extrapolating this to RIN will likely exacerbate the 


\section{Table 5. Tips and tricks for robotic intracorporeal neobladder}

\begin{tabular}{ll}
\hline Stage of operation & Tip \\
\hline $\begin{array}{l}\text { Demarcation of the } \\
\text { neobladder bowel }\end{array}$ & $\begin{array}{l}\text { Easier to bring down in women. Adhesiolysis of ileal segment, and possible division of } \\
\text { segment } \\
\text { mesentery taking care not to compromise blood supply may help to bring ileum down }\end{array}$ \\
$\begin{array}{ll}\text { Urethro-ileal anastomosis } & \text { Sub-urethral to ileal serosa Rocco type suture, to allow for tension-free urethro-ileal } \\
\text { anastomosis }\end{array}$ \\
$\begin{array}{l}\text { Isolation of neobladder } \\
\text { bowel segment and re- } \\
\text { anastomosis of the bowel }\end{array}$ & $\begin{array}{l}10 \mathrm{~cm} \text { for right ileal limb and } 40 \mathrm{~cm} \text { for left ileal limb, which will consist of } 10 \mathrm{~cm} \text { ileal } \\
\text { chimney and } 20 \mathrm{~cm} \text { left lateral ileal limb }\end{array}$
\end{tabular}

anastomosis of the bowe

Detubularisation of the To set up closure of the posterior neobladder plate and the shape of the neobladder the ileal neobladder limbs and position of 3 stay sutures is crucial

formation of the posterior Open the ileal lumen over suction tube plate

Anterior neobladder

closure

Stent insertion

Uretero-ureteric

anastomosis

Wallace uretero-ilea

anastomosis

First suture from apex of left lateral limb to base of ileal chimney allows clear approach for anterior neobladder closure

Using the seldinger technique, a venflon is inserted though the suprapubic area, and a guidewire is introduced, over which the stent is passed. Within the abdomen, a robotic needle holder is passed through the ileal chimney from top to bottom, ensuring the needle holder is closed to prevent injury to the chimney. Once it emerges from the bottom of the chimney, the guidewire is grasped, and pulled out through the tip of the chimney. The stent is passed over the guidewire and fed down the ureter. This process is repeated for right and left ureters

Both ureters are elevated in the right side of the abdomen using the fourth arm. The posterior ureteric plate is set up by the first suture, which is out to in on the lower medial side of the open right ureter, then in to out on the lower medial side of the left ureter, and then sutured. It is then brought back through the posterior surface of the right ureter from out to in. This opens up the posterior plate, so it is much easier to see the medial edges of each ureter. Finally, a running suture is passed superionly closing the posterior surface of right to left ureters

The first ileal chimney to ureteric sutures is below the stents and brings the ileal chimney at 6 o'clock to the lowest part of the uretero-ureteric anastomosis. This allows closure of right side of ileal-ureteric anastomosis. The fourth arm can then be dropped to show the left side of the uretero-ileal anastomosis, which is then closed under direct vision

Completion of neobladder Following this the rest of the neobladder is closed around the stents and inflated to test for closure

\section{Challenge and possible complication}

Figure

nability to bring the ileum into the pelvis may result in conversion to ileal conduit

Without being tension-free, the anastomosis may break down notwithstanding technical difficulty of suturing without losing its stabilizing effect

Position of bowel that is re-anastomosed within right side of abdomen, and angle of stapler determined by fourth arm trocar position is important. Re-anastomosis is done by using $60 \mathrm{~mm}$ then $45 \mathrm{~mm}$ staples, then closed with final $60 \mathrm{~mm}$ staples. Anastomotic leak is a potential complication

Opening ileal lumen over the suction tube helps prevent injury to posterior wall of the ileum. Judicious placement of 3 stay sutures limits technical difficulty of suturing posterior plate of the neobladder and eases later folding and anterior closure. Potential complications include njury to posterior ileal wall and posterior neobladder leak or rupture

Judicious suturing will prevent neobladder leak or rupture

The robotic needle holder has to pass down the ileal chimney carefully to Figure 10 prevent injury, and later the stents must be pushed into the rena collecting system. They are sutured together to the skin of the anterior abdominal wall and removed at 14 days. They allow the uretero-ureteric and uretero-ileal anastomoses to heal, and help to prevent ureteric

stricturing, anastomotic breakdown and leakage

The orientation of the suturing should be followed meticulously to allow efficient progress. Poor technique may cause anastomotic leakage and ureteric strictures

One of the most technically challenging parts of the procedures, and important at the beginning to keep sutures below the stents. Poor echnique may cause anastomotic breakdown and leakage Judicious suturing to ensure tight closure. Potential complication is neobladder leak
Figures 2 and 3

gure 4

Figures 5 and 6

Figures 11-14

Figure 18 
difference in cost. The only study to date comparing RARC with intracorporeal urinary diversion with ORC is from the UK and examined 221 patients who underwent RARC, 7.7\% of whom had RIN vs. 100 patients who had ORC, all of whom had ileal conduit ${ }^{[30]}$. They found RARC $18.9 \%$ more expensive than ORC, primarily resulting from LOS and OTs, but also influenced by the volume of cases per annul. The influence of learning curves and surgeon experience is clear, and this would seem to support centralization from an economic point of view, such that larger high-volume centers will have shorter operating times, fewer complications, and lower robotic costs per case. Although it is reasonable to assume this applies to RARC with RIN, which exacerbates all the above, further studies are necessary to evaluate specific RIN case costs.

\section{Learning curve}

Because RIN is technically challenging, it would be fair to suggest this has impacted its more widespread adoption. In an attempt to standardize the learning, a number of studies have assessed the LC of RIN, a concept that has its origins in the aviation industry and training pilots ${ }^{[31]}$. LC analysis aims to determine carefully chosen outcomes metrics to assess a learner's improvement, which in the context of RIN would include operative time, conversion rates, blood loss, complications rates, and LOS. Often studies have also included lymph node yield and surgical margin status, which may be more relevant to the EPLND and RARC. Nevertheless, embarking on learning this procedure does have advantages. Not only just the incorporeal approach, which keeps the bowel in the abdomen, reducing blood and fluid loss and hypothermia, minimizes surgical trauma and allows the bowel to recover quicker ${ }^{[5]}$ but also the inherent advantages of orthotopic neobladder with improved physical image and QoL, as well as better sexual function and continence in the majority of patients ${ }^{[4]}$. In a recent LC analysis of 167 patients, with case numbers divided into tertiles to assess the impact of increasing case number on the defined outcomes (OR time, complications, LOS), Tuderti et al. ${ }^{[32]}$ found those patients operated on in the early part of the LC had worse perioperative and functional outcomes, which then normalized in later tertiles. Similarly, examining the first 100 consecutive cases of RARC and RIN, D'Annunzio et al ${ }^{[33]}$ found an OR time plateau could be achieved at 20 cases, but 60 cases were required to achieve benchmark outcomes determined by the Pasadena RARC consensus. Earlier, Collins et al. ${ }^{[34]}$ examining a series of 67 patients that formed the first cases of a surgeon at a high-volume center, showed no compromise on perioperative and pathological outcomes. However, their study emphasized the importance of an experienced robotic mentor and team to supervise and guide the learning surgeon through these cases $^{[34]}$. This view is supported by Porreca et al ${ }^{[35]}$, and using the analogy of LC analysis and educational research that has studied Robot-Assisted Radical Prostatectomy, a modern surgical approach to RARC and RIN. This approach would be multimodal, with didactic sessions, time to examine expert videos, and a highly structured modular approach to live operating under the close supervision of a mentor in a high-volume center, which will minimize any LC impact on both oncological and functional outcomes. Further studies with longer follow-up are needed to provide evidence for this viewpoint.

\section{ERAS and RIN}

ERAS programs' benefits and detailed protocols for patients undergoing RARC are well documented ${ }^{[7]}$ and were first described for colorectal patients in the late 1990s. Kehlett outlined a multimodal protocol of activities aimed at reducing the negative physiological and biochemical effects of surgical trauma to speed up postoperative recovery ${ }^{[36]}$. Using a minimally invasive approach, RARC is synchronistic with this concept, and ERAS protocols have been beneficial ${ }^{[7]}$. However, the combination of ERAS programs and robotic ICUD and specifically RIN has been less well studied. Tan et al. ${ }^{[3]}$ demonstrated that a detailed and well-applied ERAS program combined with intracorporeal urinary diversion could significantly reduce LOS and have a synergistic benefit on perioperative metrics without affecting 90 -day complication and readmission risk, but out of 145 patients studied, only 11 underwent RIN. Similarly, Cerruto et al. ${ }^{[38]}$, in a series of 31 patients having combined RC and Neobladder, demonstrated reduced complications in the 
postoperative period, but the surgery was not performed robotically. There are a number of studies examining ERAS in RARC, but only a small number of these include RIN, and although it is perceptively that the combination would be beneficial, more study is required ${ }^{[7]}$.

\section{CONCLUSION}

There has been slow adoption of RARC with RIN in specialized centers for the treatment of high-risk and muscle-invasive bladder cancer. Although it is a challenging technique, it represents a natural evolution of the minimally invasive approach with robotics. The technique has a steep LC and longer OTs as a result and many potential advantages, such as less bowel manipulation, less fluid and blood loss, and quicker return of bowel function. Larger retrospective series with longer follow up are beginning to emerge, but there remains significant heterogeneity in the experience of the teams and surgeons involved, making meaningful interpretation of their results challenging. Nevertheless, from higher volume centers, results demonstrate the safety and feasibility of RARC with RIN. Perceptively, perioperative and functional outcomes for wellselected patients may be superior to ileal conduit and ECUD. However, the results of well-structured studies such as the iROC are keenly anticipated and will hopefully shed light on some of these unanswered questions.

\section{DECLARATIONS}

\section{Authors' contributions}

Performed data analysis, interpretation, and manuscript write up: Maqboul F

Technical description of operative steps, data acquisition: Dovey Z

Review of manuscript: Thinagaran JKR

Review and substantial contributions to conception and design of study: Dovey Z, Wiklund P

\section{Availability of data and materials}

Not applicable.

\section{Financial support and sponsorship}

None.

\section{Conflicts of interest}

Dr. Zach Dovey is Medical Director and stock owner (with certificate of shares) of Medtech Holdings Ltd.

\section{Ethical approval and consent to participate}

Not applicable.

\section{Consent for publication}

Not applicable.

\section{Copyright}

(c) The Author(s) 2021.

\section{REFERENCES}

1. Zamboni S, Soria F, Mathieu R, et al; European Association of Urology - Young Academic Urologists (EAU-YAU); Urothelial carcinoma working group. Differences in trends in the use of robot-assisted and open radical cystectomy and changes over time in peri-operative outcomes among selected centres in North America and Europe: an international multicentre collaboration. BJU Int 2019;124:656-64. DOI PubMed

2. Hussein AA, May PR, Jing Z, et al; Collaborators. Outcomes of intracorporeal urinary diversion after robot-assisted radical cystectomy: results from the international robotic cystectomy consortium. J Urol 2018;199:1302-11. DOI PubMed

3. Chan KG, Guru K, Wiklund P, et al; Pasadena Consensus Panel. Robot-assisted radical cystectomy and urinary diversion: technical recommendations from the Pasadena Consensus Panel. Eur Urol 2015;67:423-31. DOI PubMed 
4. Qu LG, Lawrentschuk N. Orthotopic neobladder reconstruction: patient selection and perspectives. Res Rep Urol 2019;11:333-41. DOI PubMed PMC

5. Hosseini A, Mortezavi A, Sjöberg S, et al. Robot-assisted intracorporeal orthotopic bladder substitution after radical cystectomy: perioperative morbidity and oncological outcomes - a single-institution experience. BJU Int 2020;126:464-71. DOI PubMed

6. Otaola-Arca H, Seetharam Bhat KR, Patel VR, Moschovas MC, Orvieto M. Totally intracorporeal robot-assisted urinary diversion for bladder cancer (part 2). Review and detailed characterization of the existing intracorporeal orthotopic ileal neobladder. Asian J Urol 2021;8:63-80. DOI PubMed PMC

7. Collins JW, Patel H, Adding C, et al. Enhanced recovery after robot-assisted radical cystectomy: EAU robotic urology section scientific working group consensus view. Eur Urol 2016;70:649-60. DOI PubMed

8. Wiklund NP, Poulakis V. Robotic neobladder. BJU Int 2011;107:1514-37. DOI PubMed

9. Chopra S, de Castro Abreu AL, Berger AK, et al. Evolution of robot-assisted orthotopic ileal neobladder formation: a step-by-step update to the University of Southern California (USC) technique. BJU Int 2017;119:185-91. DOI PubMed

10. Tan WS, Lamb BW, Kelly JD. Evolution of the neobladder: A critical review of open and intracorporeal neobladder reconstruction techniques. Scand J Urol 2016;50:95-103. DOI PubMed

11. Chan KG, Collins JW, Wiklund NP. Robot-assisted radical cystectomy: extracorporeal vs intracorporeal urinary diversion. $J$ Urol 2015;193:1467-9. DOI PubMed

12. Wilson TG, Guru K, Rosen RC, et al; Pasadena Consensus Panel. Best practices in robot-assisted radical cystectomy and urinary reconstruction: recommendations of the Pasadena Consensus Panel. Eur Urol 2015;67:363-75. DOI PubMed

13. Tuderti G, Mastroianni R, Flammia S, et al. Sex-sparing robot-assisted radical cystectomy with intracorporeal Padua ileal neobladder in female: surgical technique, perioperative, oncologic and functional outcomes. J Clin Med 2020;9:577. DOI PubMed PMC

14. Hautmann RE, de Petriconi RC, Volkmer BG. Lessons learned from 1,000 neobladders: the 90-day complication rate. J Urol 2010;184:990-4; quiz 1235. DOI PubMed

15. Nguyen DP, Al Hussein Al Awamlh B, Wu X, et al. Recurrence patterns after open and robot-assisted radical cystectomy for bladder cancer. Eur Urol 2015;68:399-405. DOI PubMed PMC

16. Simone G, Tuderti G, Misuraca L, et al. Perioperative and mid-term oncologic outcomes of robotic assisted radical cystectomy with totally intracorporeal neobladder: results of a propensity score matched comparison with open cohort from a single-centre series. Eur $J$ Surg Oncol 2018;44:1432-8. DOI PubMed

17. Martin AS, Corcoran AT. Contemporary techniques and outcomes of robotic assisted radical cystectomy with intracorporeal urinary diversion. Transl Androl Urol 2021;10:2216-32. DOI PubMed PMC

18. Stein JP, Lieskovsky G, Cote R, et al. Radical cystectomy in the treatment of invasive bladder cancer: long-term results in 1,054 patients. J Clin Oncol 2001;19:666-75. DOI PubMed

19. Hussein AA, Elsayed AS, Aldhaam NA, et al. Ten-year oncologic outcomes following robot-assisted radical cystectomy: results from the international robotic cystectomy consortium. J Urol 2019;202:927-35. DOI PubMed

20. Obrecht F, Youssef NA, Burkhardt O, et al. Robot-assisted radical cystectomy and intracorporeal orthotopic neobladder: 1-year functional outcomes. Asian J Androl 2020;22:145-8. DOI PubMed PMC

21. Satkunasivam R, Santomauro M, Chopra S, et al. Robotic intracorporeal orthotopic neobladder: urodynamic outcomes, urinary function, and Health-related Quality of Life. Eur Urol 2016;69:247-53. DOI PubMed

22. Yadav SS, Gangkak G, Mathur R, Yadav RG, Tomar V. Long-term functional, urodynamic, and metabolic outcome of a modified orthotopic neobladder created with a short ileal segment: our 5-year experience. Urology 2016;94:167-72. DOI PubMed

23. Jonsson MN, Adding LC, Hosseini A, et al. Robot-assisted radical cystectomy with intracorporeal urinary diversion in patients with transitional cell carcinoma of the bladder. Eur Urol 2011;60:1066-73. DOI PubMed

24. Parekh DJ, Reis IM, Castle EP, et al. Robot-assisted radical cystectomy versus open radical cystectomy in patients with bladder cancer (RAZOR): an open-label, randomised, phase 3, non-inferiority trial. Lancet 2018;391:2525-36. DOI PubMed

25. Mastroianni R, Tuderti G, Anceschi U, et al. Comparison of patient-reported Health-related Quality of Life between open radical cystectomy and robot-assisted radical cystectomy with intracorporeal urinary diversion: interim analysis of a randomised controlled trial. Eur Urol Focus 2021:S2405-4569(21)00059. DOI PubMed

26. Simone G, Papalia R, Leonardo C, et al. Prostatic capsule and seminal vesicle-sparing cystectomy: improved functional results, inferior oncologic outcome. Urology 2008;72:162-6. DOI PubMed

27. Catto JWF, Khetrapal P, Ambler G, et al. Robot-assisted radical cystectomy with intracorporeal urinary diversion versus open radical cystectomy (iROC): protocol for a randomised controlled trial with internal feasibility study. BMJ Open 2018;8:e020500. DOI PubMed PMC

28. Morii Y, Osawa T, Suzuki T, et al. Cost comparison between open radical cystectomy, laparoscopic radical cystectomy, and robotassisted radical cystectomy for patients with bladder cancer: a systematic review of segmental costs. BMC Urol 2019;19:110. DOI PubMed PMC

29. Lee R, Ng CK, Shariat SF, et al. The economics of robotic cystectomy: cost comparison of open versus robotic cystectomy. BJU Int 2011;108:1886-92. DOI PubMed

30. Bansal SS, Dogra T, Smith PW, et al. Cost analysis of open radical cystectomy versus robot-assisted radical cystectomy. BJU Int 2018;121:437-44. DOI PubMed

31. Wright TP. Factors affecting the cost of airplanes. Journal of the Aeronautical Sciences 1936;3:122-8. DOI PubMed

32. Tuderti G, Mastroianni R, Brassetti A, et al. Robot-assisted radical cystectomy with intracorporeal neobladder: impact of learning curve and long-term assessment of functional outcomes. Minerva Urol Nefrol 2020. DOI PubMed 
33. D'annunzio S, Lombardo R, Mastroianni R, et al. Benchmarking pasadena consensus along the learning curve of robotic radical ystectomy with intracorporeal neobladder: cusum based assessment. Eur Urol Open Sci 2020;20:S172. DOI

34. Collins JW, Tyritzis S, Nyberg T, et al. Robot-assisted radical cystectomy (RARC) with intracorporeal neobladder - what is the effect of the learning curve on outcomes? BJU Int 2014;113:100-7. DOI PubMed

35. Porreca A, Mineo Bianchi F, Romagnoli D, et al. Robot-assisted radical cystectomy with totally intracorporeal urinary diversion: surgical and early functional outcomes through the learning curve in a single high-volume center. J Robot Surg 2020;14:261-9. DOI PubMed

36. Kehlet H. Multimodal approach to control postoperative pathophysiology and rehabilitation. Br J Anaesth 1997;78:606-17. DOI PubMed

37. Tan WS, Tan MY, Lamb BW, et al. Intracorporeal robot-assisted radical cystectomy, together with an enhanced recovery programme, improves postoperative outcomes by aggregating marginal gains. BJU Int 2018;121:632-9. DOI PubMed

38. Cerruto MA, De Marco V, D'Elia C, et al. Introduction of an enhanced recovery protocol to reduce short-term complications following radical cystectomy and intestinal urinary diversion with vescica ileale Padovana neobladder. Urol Int 2014;92:35-40. DOI PubMed

39. Collins JW, Sooriakumaran P, Sanchez-Salas R, et al. Robot-assisted radical cystectomy with intracorporeal neobladder diversion: The Karolinska experience. Indian J Urol 2014;30:307-13. DOI PubMed PMC

40. Goh AC, Gill IS, Lee DJ, et al. Robotic intracorporeal orthotopic ileal neobladder: replicating open surgical principles. Eur Urol 2012;62:891-901. DOI PubMed

41. Tyritzis SI, Hosseini A, Collins J, et al. Oncologic, functional, and complications outcomes of robot-assisted radical cystectomy with totally intracorporeal neobladder diversion. Eur Urol 2013;64:734-41. DOI PubMed

42. Desai MM, Gill IS, de Castro Abreu AL, et al. Robotic intracorporeal orthotopic neobladder during radical cystectomy in 132 patients. J Urol 2014;192:1734-40. DOI PubMed

43. Schwentner C, Sim A, Balbay MD, et al. Robot-assisted radical cystectomy and intracorporeal neobladder formation: on the way to a standardized procedure. World J Surg Oncol 2015;13:3. DOI PubMed PMC

44. Gu Q, Xia J, Xu A, Zhang T, Wang Z. Robot-assisted radical cystectomy with totally intracorporeal neobladder diversion: perioperative, oncologic, and functional outcomes. Transl Androl Urol 2020;9:2606-15. DOI PubMed PMC 